\title{
Analysis of a Coaxial Waveguide with Finite-Length Impedance Loadings in the Inner and Outer Conductors
}

\author{
Feray Hacivelioğlu and Alinur Büyükaksoy \\ Department of Mathematics, Gebze Institute of Technology, P.O. Box. 141, Gebze, 41400 Kocaeli, Turkey \\ Correspondence should be addressed to Alinur Büyükaksoy, alinur@gyte.edu.tr
}

Received 30 November 2008; Revised 2 March 2009; Accepted 10 May 2009

Recommended by Mohammad Younis

\begin{abstract}
A rigorous Wiener-Hopf approach is used to investigate the band stop filter characteristics of a coaxial waveguide with finite-length impedance loading. The representation of the solution to the boundary-value problem in terms of Fourier integrals leads to two simultaneous modified WienerHopf equations whose formal solution is obtained by using the factorization and decomposition procedures. The solution involves 16 infinite sets of unknown coefficients satisfying 16 infinite systems of linear algebraic equations. These systems are solved numerically and some graphical results showing the influence of the spacing between the coaxial cylinders, the surface impedances, and the length of the impedance loadings on the reflection coefficient are presented.
\end{abstract}

Copyright (C) 2009 F. Hacıvelioğlu and A. Büyükaksoy. This is an open access article distributed under the Creative Commons Attribution License, which permits unrestricted use, distribution, and reproduction in any medium, provided the original work is properly cited.

\section{Introduction}

Coaxial discontinuity structures are widely used as an element of microwave devices, and in the permeability and permittivity measurement for materials [1-3]. Hence, the diffraction at discontinuities in coaxial waveguides is a very important topic in microwave theory and have been subjected to numerous past investigations. Most simple types of discontinuities such as steps in inner or outer conductors (see, e.g., [4-7]) and wall impedance discontinuities [8,9] were analyzed and characterized. For example, in $[8,9]$ the scattering of a shielded surface wave in a coaxial waveguide by a wall impedance discontinuity in the inner cylinder has been analyzed. These classical results are related mostly with isolated discontinuities, and fail when there are several of them close enough to interfere with each other. The aim of the present work is to consider a new canonical scattering problem consisting of the propagation of the dominant TEM mode at the finite-length impedance discontinuities in the inner and outer conductors of a coaxial waveguide (see Figure 1). The contributions from the successive 
impedance discontinuities are accounted for through the solution of a system of coupled modified Wiener-Hopf equations. Notice that the present problem may also be thought as a first-order approximation for coaxial waveguides loaded with a dielectric filled shallow grooves, forming a band stop filter in the microwave region [10]. The band stop properties of such structures are exploited in multiple frequency circuits, such as parametric amplifiers. Constant surface impedance model may also be used to simulate corrugations, thin finitelength dielectric linings on the inner and outer metallic walls of a coaxial waveguide [11-13]. The impedance representation is also applicable to ribbed or helical slow-wave structures if the repeat distance of the ribs or helix is small relative to the distance within which the field in the guide changes substantially. Hence the present problem is also of interest when a coaxial phase shifter is used. It consists of a section of coaxial waveguide with a helix cut in the inner rod, which can be screwed to a variable depth into the end of the smooth inner conductor. The length of this screwed section then provides the required phase shift [9].

A problem similar to the one considered in this work has been recently treated by the authors in the simpler case where the impedance loading is present on the outer conductor only [14]. The generalization consisting of assuming that both inner and outer conductors are loaded with different surface impedances of finite-length is not straightforward since we end up with a coupled system of modified Wiener-Hopf equations. It is well known that the classical Wiener-Hopf technique is applicable when the diffracting obstacle is infinitely thin and has a semi-infinite straight boundary. However, since the impedance loadings on the inner and outer conductors of the coaxial waveguide are of finite-length and are different from each other, the resulting Wiener-Hopf equations are modified and coupled, respectively [15].

The method adopted here is similar to that described in [16] where parallel plate waveguides with different impedance loading of finite-length on the upper and lower plates were dealt with. Indeed, by using the analytical properties of the functions that occur we were able to uncouple these modified Wiener-Hopf equations and obtain the exact formal solution through the factorization and decomposition procedures. The formal solution involves 8 sets of infinitely many constants satisfying 8 sets of infinite systems of algebraic equations. An approximate solution to these infinite system of equations is obtained numerically and the radiation characteristics of the impedance-loaded coaxial waveguide is studied.

\section{Analysis}

Consider a coaxial waveguide whose inner cylinder is of radius $\rho=a$, while the radius of the outer cylinder is $\rho=b$ with $(\rho, \phi, z)$ being the usual cylindrical coordinates. The part $0<z<l$ of the inner and outer conductors are characterized by different constant surface impedances denoted by $Z_{1}=\eta_{1} Z_{0}$ and $Z_{2}=\eta_{2} Z_{0}$, respectively, with $Z_{0}$ being the characteristic impedance of the free space.

Let the incident TEM mode with angular frequency $\omega$ and propagating in the positive $z$ direction be given by $H_{\rho}^{i}=0, H_{\phi}^{i}=u^{i}, H_{z}^{i}=0$, with

$$
u^{i}=\frac{\exp (i k z)}{\rho}
$$




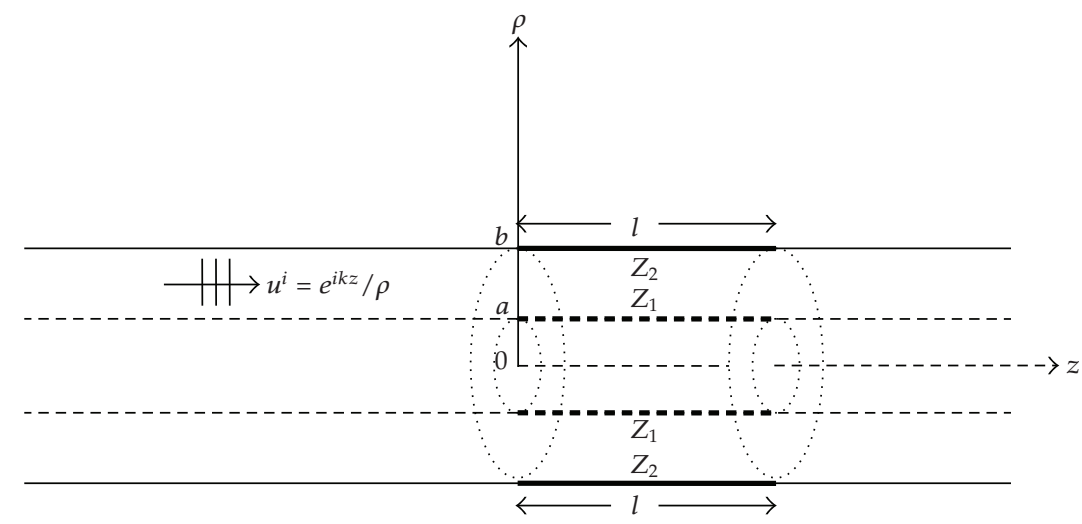

Figure 1: Coaxial cable with a finite-length impedance loading in the inner and outer conductors.

where an $\exp (-i \omega t)$ time factor is assumed and suppressed. $k$ is the propagation constant which is assumed to have a small imaginary part corresponding to a medium with damping. The lossless case can be obtained by letting $\operatorname{Im} k \rightarrow 0$ at the end of the analysis.

\subsection{Formulation of the Problem}

The total field $u^{T}(\rho, z)$ can be written as

$$
u^{T}=u^{i}+u_{1}(\rho, z), \quad \rho \in(a, b), z \in(-\infty, \infty),
$$

$u_{1}(\rho, z)$ appearing in $(2.1 \mathrm{~b})$ is an unknown function which satisfies the Helmholtz equation

$$
\left(\frac{1}{\rho} \frac{\partial}{\partial \rho}\left(\rho \frac{\partial}{\partial \rho}\right)+\frac{\partial^{2}}{\partial z^{2}}+k^{2}-\frac{1}{\rho^{2}}\right) u_{1}(\rho, z)=0, \quad \rho \in(a, b), z \in(-\infty, \infty)
$$

and the following boundary conditions and continuity relations:

$$
\begin{aligned}
& u_{1}(b, z)+b \frac{\partial}{\partial \rho} u_{1}(b, z)=0, \quad z \in(-\infty, 0) \cup(l, \infty), \\
& u_{1}(a, z)+a \frac{\partial}{\partial \rho} u_{1}(a, z)=0, \quad z \in(-\infty, 0) \cup(l, \infty), \\
& \eta_{2}^{*} u_{1}(b, z)-\frac{1}{i k} \frac{\partial}{\partial \rho} u_{1}(b, z)=-\frac{\eta_{2}}{b} e^{i k z}, \quad z \in(0, l), \\
& \eta_{1}^{*} u_{1}(a, z)+\frac{1}{i k} \frac{\partial}{\partial \rho} u_{1}(a, z)=-\frac{\eta_{1}}{a} e^{i k z}, \quad z \in(0, l),
\end{aligned}
$$


where

$$
\begin{aligned}
& \eta_{1}^{*}=\eta_{1}+\frac{1}{i k a}, \\
& \eta_{2}^{*}=\eta_{2}-\frac{1}{i k b} .
\end{aligned}
$$

To obtain the unique solution to the mixed boundary value problem stated by (2.2) and (2.3a)-(2.3d) one has to take into account the following edge and radiation conditions [17]:

$$
\begin{gathered}
\begin{array}{l}
u^{T}(a, z) \\
u^{T}(b, z)
\end{array}= \begin{cases}\mathcal{O}\left(|z|^{1 / 2}\right), & |z| \longrightarrow 0 \\
\mathcal{O}\left(|z-l|^{1 / 2}\right), & z \longrightarrow l\end{cases} \\
u_{1}(\rho, z)=\mathcal{O}\left(e^{i k|z|}\right), \quad|z| \longrightarrow \infty
\end{gathered}
$$

The infinite-range Fourier transform of (2.2) yields

$$
\left(\frac{1}{\rho} \frac{\partial}{\partial \rho}\left(\rho \frac{\partial}{\partial \rho}\right)+K^{2}(\alpha)-\frac{1}{\rho^{2}}\right) F(\rho, \alpha)=0
$$

with

$$
\begin{gathered}
F(\rho, \alpha)=F^{-}(\rho, \alpha)+F_{1}(\rho, \alpha)+e^{i \alpha l} F^{+}(\rho, \alpha), \\
F^{-}(\rho, \alpha)=\int_{-\infty}^{0} u_{1}(\rho, \alpha) e^{i \alpha z} d z, \\
F_{1}(\rho, \alpha)=\int_{0}^{l} u_{1}(\rho, \alpha) e^{i \alpha z} d z, \\
F^{+}(\rho, \alpha)=\int_{l}^{\infty} u_{1}(\rho, \alpha) e^{i \alpha(z-l)} d z .
\end{gathered}
$$

In $(2.4 a), K(\alpha)$ stands for

$$
K(\alpha)=\sqrt{k^{2}-\alpha^{2}}
$$

The square-root function is defined in the complex $\alpha$-plane, cut along $\alpha=k$ to $\alpha=k+i \infty$ and $\alpha=-k$ to $\alpha=-k-i \infty$, such that $K(0)=k$ (see Figure 2). 


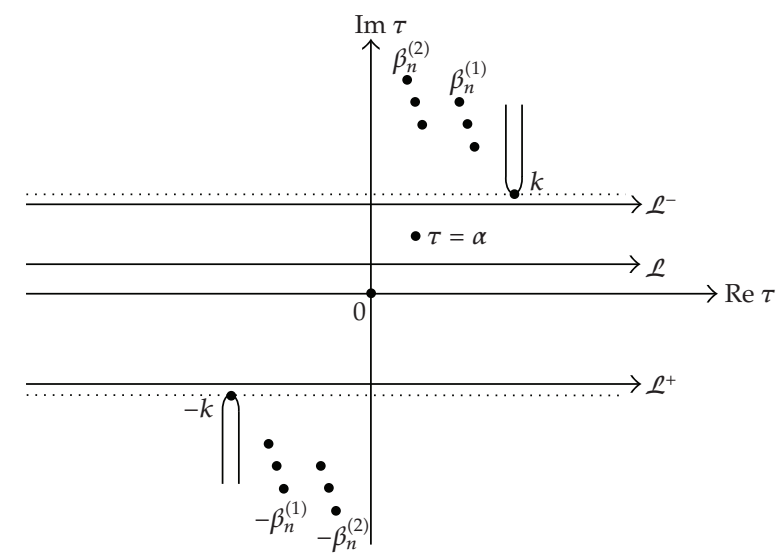

Figure 2: Branch-cuts and integration lines in the complex plane.

Owing to the analytical properties of Fourier integrals, $F^{+}(\rho, \alpha)$ and $F^{-}(\rho, \alpha)$ are yet unknown functions which are regular in the half-planes $\operatorname{Im}(\alpha)>\operatorname{Im}(-k)$ and $\operatorname{Im}(\alpha)<\operatorname{Im}(k)$, respectively. The function $F_{1}(\rho, \alpha)$ defined by $(2.4 \mathrm{~d})$ is an unknown entire function.

The solution of $(2.4 \mathrm{a})$ reads

$$
F^{-}(\rho, \alpha)+F_{1}(\rho, \alpha)+e^{i \alpha l} F^{+}(\rho, \alpha)=A(\alpha) J_{1}(K \rho)+B(\alpha) Y_{1}(K \rho) .
$$

Here $A(\alpha)$ and $B(\alpha)$ are spectral coefficients to be determined. By using the Fourier transform of the boundary conditions in (2.3a) and (2.3b), one can easily show that they are related to $P_{1}(a, \alpha)$ and $P_{1}(b, \alpha)$ through

$$
\left[\begin{array}{c}
A(\alpha) \\
B(\alpha)
\end{array}\right]=\left[\begin{array}{cc}
-\frac{K(\alpha) Y_{0}(K b)}{a T_{1}(a, b, \alpha)} & \frac{K(\alpha) Y_{0}(K a)}{b T_{1}(a, b, \alpha)} \\
\frac{K(\alpha) J_{0}(K b)}{a T_{1}(a, b, \alpha)} & -\frac{K(\alpha) J_{0}(K a)}{b T_{1}(a, b, \alpha)}
\end{array}\right]\left[\begin{array}{l}
P_{1}(a, \alpha) \\
P_{1}(b, \alpha)
\end{array}\right]
$$

with $P_{1}(\rho, \alpha)$ being given by

$$
P_{1}(\rho, \alpha)=F_{1}(\rho, \alpha)+\rho \dot{F}_{1}(\rho, \alpha)
$$

In (2.7b), the $\operatorname{dot}(\cdot)$ denotes the derivative with respect to $\rho$, that is, $\dot{F}_{1}(\rho, \alpha)=(\partial / \partial \rho) F_{1}(\rho, \alpha)$. Hence, (2.6) can be rearranged as

$$
F^{-}(\rho, \alpha)+F_{1}(\rho, \alpha)+e^{i \alpha l} F^{+}(\rho, \alpha)=\frac{T_{2}(\rho, b, \alpha)}{a T_{1}(a, b, \alpha)} P_{1}(a, \alpha)-\frac{T_{2}(\rho, a, \alpha)}{b T_{1}(a, b, \alpha)} P_{1}(b, \alpha)
$$


with

$$
\begin{gathered}
T_{1}(a, b, \alpha)=K^{2}(\alpha)\left[J_{0}(K a) Y_{0}(K b)-J_{0}(K b) Y_{0}(K a)\right] \\
T_{2}(a, b, \alpha)=K(\alpha)\left[J_{1}(K a) Y_{0}(K b)-J_{0}(K b) Y_{1}(K a)\right]
\end{gathered}
$$

Multiplying both sides of (2.3c) and (2.3d) by $e^{i \alpha z}$ and then integrating from 0 to $l$ we obtain

$$
\begin{aligned}
& \eta_{2}^{*} F_{1}(b, \alpha)=-\frac{\eta_{2}}{i b(k+\alpha)}\left[e^{i(k+\alpha) l}-1\right]+\frac{1}{i k} \dot{F}_{1}(b, \alpha) \\
& \eta_{1}^{*} F_{1}(a, \alpha)=-\frac{\eta_{1}}{i a(k+\alpha)}\left[e^{i(k+\alpha) l}-1\right]-\frac{1}{i k} \dot{F}_{1}(a, \alpha) .
\end{aligned}
$$

Putting $\rho=b$ and then $\rho=a$ in (2.8a) and its derivative with respect to $\rho$, and then using the relations (2.9) we end up with the following coupled systems of modified Wiener-Hopf equations valid in the strip $\operatorname{Im}(-k)<\operatorname{Im}(\alpha)<\operatorname{Im}(k)$ :

$$
\begin{aligned}
& i k a \Psi_{1}^{-}(a, \alpha)+V_{1}(\alpha) P_{1}(a, \alpha)-i k a e^{i \alpha l} \Psi_{1}^{+}(a, \alpha)=\frac{2 i k \eta_{1}}{\pi b T_{1}(a, b, \alpha)} P_{1}(b, \alpha)-k \eta_{1}\left[\frac{e^{i(k+\alpha) l}-1}{k+\alpha}\right] \\
& i k b \Psi_{2}^{-}(b, \alpha)-V_{2}(\alpha) P_{1}(b, \alpha)-i k b e^{i \alpha l} \Psi_{2}^{+}(b, \alpha)=\frac{-2 i k \eta_{2}}{\pi a T_{1}(a, b, \alpha)} P_{1}(a, \alpha)-k \eta_{2}\left[\frac{e^{i(k+\alpha) l}-1}{k+\alpha}\right]
\end{aligned}
$$

with,

$$
\begin{gathered}
V_{1}(\alpha)=\frac{x_{1}(\alpha)}{T_{1}(a, b, \alpha)}, \\
V_{2}(\alpha)=\frac{x_{2}(\alpha)}{T_{1}(a, b, \alpha)}, \\
x_{1}(\alpha)=T_{1}(a, b, \alpha)+i k \eta_{1} T_{2}(a, b, \alpha), \\
x_{2}(\alpha)=T_{1}(a, b, \alpha)+i k \eta_{2} T_{2}(b, a, \alpha), \\
\Psi_{1}^{-}(\alpha)=\eta_{1}^{*} F_{-}(a, \alpha)+\frac{1}{i k} \dot{F}_{-}(a, \alpha), \\
\Psi_{1}^{+}(\alpha)=\eta_{1}^{*} F_{+}(a, \alpha)+\frac{1}{i k} \dot{F}_{+}(a, \alpha), \\
\Psi_{2}^{-}(\alpha)=\eta_{2}^{*} F_{-}(b, \alpha)-\frac{1}{i k} \dot{F}_{-}(b, \alpha), \\
\Psi_{2}^{+}(\alpha)=\eta_{2}^{*} F_{+}(a, \alpha)-\frac{1}{i k} \dot{F}_{+}(b, \alpha) .
\end{gathered}
$$




\subsection{Solution of the Wiener-Hopf Equations}

Now, let us rearrange the equations in (2.10) as

$$
\begin{aligned}
& R_{1 *}^{-}(\alpha)-V_{1}(\alpha) P_{1}(a, \alpha)+e^{i \alpha l} S_{1}^{+}(\alpha)=-\frac{2 i k \eta_{1}}{\pi b T_{1}(a, b, \alpha)} P_{1}(b, \alpha) \\
& R_{2 *}^{-}(\alpha)+V_{2}(\alpha) P_{1}(b, \alpha)+e^{i \alpha l} S_{2}^{+}(\alpha)=\frac{2 i k \eta_{2}}{\pi a T_{1}(a, b, \alpha)} P_{1}(a, \alpha)
\end{aligned}
$$

with

$$
\begin{gathered}
R_{1 *}^{-}(\alpha)=\operatorname{aik} \Psi_{1}^{-}(\alpha)+\frac{k \eta_{1}}{(k+\alpha)}, \\
R_{2 *}^{-}(\alpha)=b i k \Psi_{2}^{-}(\alpha)+\frac{k \eta_{2}}{(k+\alpha)}, \\
S_{1}^{+}(\alpha)=\operatorname{aik} \Psi_{1}^{+}(\alpha)-\frac{k \eta_{1}}{(k+\alpha)} e^{i k l}, \\
S_{2}^{+}(\alpha)=b i k \Psi_{2}^{+}(\alpha)-\frac{k \eta_{2}}{(k+\alpha)} e^{i k l} .
\end{gathered}
$$

Notice that $R_{1 *}^{-}(\alpha)$ and $R_{2 *}^{-}(\alpha)$ are regular functions of $\alpha$ in the lower half-plane $\operatorname{Im}(\alpha)<\operatorname{Im}(k)$ except at the pole singularity occurring at $\alpha=-k$, while $S_{1}^{+}(\alpha)$ and $S_{2}^{+}(\alpha)$ are regular in the upper half-plane $\operatorname{Im}(\alpha)>\operatorname{Im}(-k)$.

Now, consider the Wiener-Hopf factorization of the kernel functions $V_{1,2}(\alpha)$, defined in (2.11a) as

$$
V_{1,2}(\alpha)=V_{1,2}^{+}(\alpha) V_{1,2}^{-}(\alpha)
$$

where $V_{1,2}^{+}(\alpha)$ and $V_{1,2}^{-}(\alpha)$ denote certain functions which are regular and free of zeros in the half-planes $\operatorname{Im}(\alpha)>\operatorname{Im}(-k)$ and $\operatorname{Im}(\alpha)<\operatorname{Im}(k)$, respectively. Their explicit expressions can easily be found by using the method described in [18]

$$
V_{1,2}^{ \pm}(\alpha)=\sqrt{V_{1,2}(0)} \frac{1}{\left(1 \mp \alpha / \xi_{1}\right)} \prod_{m=1}^{\infty}\left(\frac{1 \mp \alpha / \beta_{m}^{(1,2)}}{\left(1 \mp \alpha / \xi_{m+1}\right)}\right) .
$$


Here $\pm \beta_{m}^{(1)}$ and $\pm \beta_{m}^{(2)}, m=1,2, \ldots$, denote the symmetrical zeros of $V_{1}(\alpha)$ and $V_{2}(\alpha)$ :

$$
V_{1,2}\left( \pm \beta_{m}^{(1,2)}\right)=0, \quad \operatorname{Im} \beta_{m}^{(1,2)}>\operatorname{Im} k
$$

whereas $\pm \xi_{m}, m=1,2, \ldots$ are the zeros of $T_{1}(a, b, \alpha)$, that is,

$$
T_{1}\left(a, b, \pm \xi_{m}\right)=0, \quad \operatorname{Im} \xi_{m}>\operatorname{Im} k
$$

Multiplying both sides of $(2.12 \mathrm{a})$ by $1 / V_{1}^{-}(\alpha)$ and $e^{-i \alpha l} / V_{1}^{+}(\alpha)$, and both sides of (2.12b) by $1 / V_{2}^{-}(\alpha)$ and $e^{-i \alpha l} / V_{2}^{+}(\alpha)$, we obtain, after using the Wiener-Hopf decomposition procedure, the analytical continuation principle, and the Liouville theorem, the following results [19]:

$$
\begin{aligned}
& \frac{R_{1 *}^{-}(\alpha)}{V_{1}^{-}(\alpha)}-\frac{1}{2 \pi i} \int_{\mathcal{L}^{-}} \frac{S_{1}^{+}(\tau) e^{i}(\tau l d \tau}{V_{1}^{-}(\tau)(\tau-\alpha)}-\frac{2 i k \eta_{1}}{\pi b} \frac{1}{2 \pi i} \int_{\mathcal{L}^{-}} \frac{P_{1}(b, \tau) d \tau}{T_{1}(a, b, \tau) V_{1}^{-}(\tau)(\tau-\alpha)}=\frac{k \eta_{1}}{(k+\alpha) V_{1}^{+}(k)}, \\
& V_{1}^{+}(\alpha) P_{1}(a, \alpha)-\frac{1}{2 \pi i} \int_{\mathcal{L}^{+}} \frac{S_{1}^{+}(\tau) e^{i \tau l} d \tau}{V_{1}^{-}(\tau)(\tau-\alpha)}-\frac{2 i k \eta_{1}}{\pi b} \frac{1}{2 \pi i} \int_{\mathcal{L}^{+}} \frac{P_{1}(b, \tau) d \tau}{T_{1}(a, b, \tau) V_{1}^{-}(\tau)(\tau-\alpha)}=\frac{k \eta_{1}}{(k+\alpha) V_{1}^{+}(k)}, \\
& \frac{R_{2 *}^{-}(\alpha)}{V_{2}^{-}(\alpha)}-\frac{1}{2 \pi i} \int_{\mathcal{L}^{-}} \frac{S_{2}^{+}(\tau) e^{i}{ }^{-}(\tau)(\tau-\alpha)}{V^{-}(\tau-\alpha)}+\frac{2 i k \eta_{2}}{\pi a} \frac{1}{2 \pi i} \int_{\mathcal{L}^{-}} \frac{P_{1}(a, \tau) d \tau}{T_{1}(a, b, \tau) V_{2}^{-}(\tau)(\tau-\alpha)}=\frac{k \eta_{2}}{(k+\alpha) V_{2}^{+}(k)}, \\
& -V_{2}^{+}(\alpha) P_{1}(b, \alpha)-\frac{1}{2 \pi i} \int_{\mathcal{L}^{+}} \frac{S_{2}^{+}(\tau) e^{i \tau l} d \tau}{V_{2}^{-}(\tau)(\tau-\alpha)}+\frac{2 i k \eta_{2}}{\pi a} \frac{1}{2 \pi i} \int_{\mathcal{L}^{+}} \frac{P_{1}(a, \tau) d \tau}{T_{1}(a, b, \tau) V_{2}^{-}(\tau)(\tau-\alpha)}=\frac{k \eta_{2}}{(k+\alpha) V_{2}^{+}(k)}, \\
& \frac{S_{1}^{+}(\alpha)}{V_{1}^{+}(\alpha)}=-\frac{1}{2 \pi i} \int_{\mathcal{L}^{+}} \frac{R_{1 *}^{-}(\tau) e^{-i \tau l} d \tau}{V_{1}^{+}(\tau)(\tau-\alpha)}-\frac{2 i k \eta_{1}}{\pi b} \frac{1}{2 \pi i} \int_{\mathcal{L}^{+}} \frac{P_{1}(b, \tau) e^{-i \tau l} d \tau}{T_{1}(a, b, \tau) V_{1}^{+}(\tau)(\tau-\alpha)}, \\
& V_{1}^{-}(\alpha) e^{-i \tau l} P_{1}(a, \alpha)=-\frac{1}{2 \pi i} \int_{\mathcal{L}^{-}} \frac{R_{1 *}^{-}(\tau) e^{-i \tau l} d \tau}{V_{1}^{+}(\tau)(\tau-\alpha)}-\frac{2 i k \eta_{1}}{\pi b} \frac{1}{2 \pi i} \int_{\mathcal{L}^{-}} \frac{P_{1}(b, \tau) e^{-i \tau l} d \tau}{T_{1}(a, b, \tau) V_{1}^{+}(\tau)(\tau-\alpha)}, \\
& \frac{S_{2}^{+}(\alpha)}{V_{2}^{+}(\alpha)}=-\frac{1}{2 \pi i} \int_{\mathcal{L}^{+}} \frac{R_{2}^{*}(\tau) e^{-i \tau l} d \tau}{V_{2}^{+}(\tau)(\tau-\alpha)}+\frac{2 i k \eta_{2}}{\pi a} \frac{1}{2 \pi i} \int_{\mathcal{L}^{+}} \frac{P_{1}(a, \tau) e^{-i \tau l} d \tau}{T_{1}(a, b, \tau) V_{2}^{+}(\tau)(\tau-\alpha)}, \\
& -V_{2}^{-}(\alpha) e^{-i \tau l} P_{1}(b, \alpha)=\frac{1}{2 \pi i} \int_{\mathcal{L}^{-}} \frac{R_{2 *}^{-}(\tau) e^{-i \tau l} d \tau}{V_{2}^{+}(\tau)(\tau-\alpha)}+\frac{2 i k \eta_{2}}{\pi a} \frac{1}{2 \pi i} \int_{\mathcal{L}^{-}} \frac{P_{1}(a, \tau) e^{-i \tau l} d \tau}{T_{1}(a, b, \tau) V_{2}^{+}(\tau)(\tau-\alpha)}
\end{aligned}
$$

The positions of the integration lines $\mathcal{L}^{ \pm}$are shown in Figure 2. 
The above integrals can be evaluated by using Jordan's Lemma and the residue theorem. The result is

$$
\frac{R_{1 *}^{-}(\alpha)}{V_{1}^{-}(\alpha)}-\frac{2 i k \eta_{1}}{\pi b} \sum_{m=1}^{\infty} \frac{V_{1}^{+}\left(\beta_{m}^{(1)}\right) P_{1}\left(b, \beta_{m}^{(1)}\right)}{X_{1}^{\prime}\left(\beta_{m}^{(1)}\right)\left(\beta_{m}^{(1)}-\alpha\right)}-\sum_{m=1}^{\infty} \frac{V_{1}^{+}\left(\beta_{m}^{(1)}\right) e^{i \beta_{m}^{(1)} l} S_{1}^{+}\left(\beta_{m}^{(1)}\right)}{V_{1}^{\prime}\left(\beta_{m}^{(1)}\right)\left(\beta_{m}^{(1)}-\alpha\right)}=\frac{i k \eta_{1}}{i(k+\alpha) V_{1}^{+}(k)}
$$

$$
\frac{R_{2 *}^{-}(\alpha)}{V_{2}^{-}(\alpha)}+\frac{2 i k \eta_{2}}{\pi a} \sum_{m=1}^{\infty} \frac{V_{2}^{+}\left(\beta_{m}^{(2)}\right) P_{1}\left(a, \beta_{m}^{(2)}\right)}{X_{2}^{\prime}\left(\beta_{m}^{(2)}\right)\left(\beta_{m}^{(2)}-\alpha\right)}-\sum_{m=1}^{\infty} \frac{V_{2}^{+}\left(\beta_{m}^{(2)}\right) e^{i \beta_{m}^{(2)} l} S_{2}^{+}\left(\beta_{m}^{(2)}\right)}{V_{2}^{\prime}\left(\beta_{m}^{(2)}\right)\left(\beta_{m}^{(2)}-\alpha\right)}=\frac{i k \eta_{2}}{i(k+\alpha) V_{2}^{+}(k)}
$$

$$
\frac{S_{1}^{+}(\alpha)}{V_{1}^{+}(\alpha)}-\frac{2 i k \eta_{1}}{\pi b} \sum_{m=1}^{\infty} \frac{V_{1}^{+}\left(\beta_{m}^{(1)}\right) e^{i \beta_{m}^{(1)} l} P_{1}\left(b,-\beta_{m}^{(1)}\right)}{X_{1}^{\prime}\left(\beta_{m}^{(1)}\right)\left(\beta_{m}^{(1)}+\alpha\right)}-\sum_{m=1}^{\infty} \frac{V_{1}^{+}\left(\beta_{m}^{(1)}\right) e^{i \beta_{m}^{(1)} l} R_{1 *}^{-}\left(-\beta_{m}^{(1)}\right)}{V_{1}^{\prime}\left(\beta_{m}^{(1)}\right)\left(\beta_{m}^{(1)}+\alpha\right)}=0,
$$

$$
\frac{S_{2}^{+}(\alpha)}{V_{2}^{+}(\alpha)}+\frac{2 i k \eta_{2}}{\pi a} \sum_{m=1}^{\infty} \frac{V_{2}^{+}\left(\beta_{m}^{(2)}\right) e^{i \beta_{m}^{(2)} l} P_{1}\left(a,-\beta_{m}^{(2)}\right)}{X_{2}^{\prime}\left(\beta_{m}^{(2)}\right)\left(\beta_{m}^{(2)}+\alpha\right)}-\sum_{m=1}^{\infty} \frac{V_{2}^{+}\left(\beta_{m}^{(2)}\right) e^{i \beta_{m}^{(2)} l} R_{2}^{*}\left(-\beta_{m}^{(2)}\right)}{V_{2}^{\prime}\left(\beta_{m}^{(2)}\right)\left(\beta_{m}^{(2)}+\alpha\right)}=0
$$

$$
\begin{aligned}
-\frac{2 i k \eta_{1}}{\pi b} \sum_{m=1}^{\infty} \frac{V_{1}^{+}\left(\beta_{m}^{(1)}\right) P_{1}\left(b, \beta_{m}^{(1)}\right)}{X_{1}^{\prime}\left(\beta_{m}^{(1)}\right)\left(\beta_{m}^{(1)}-\alpha\right)}-\frac{2 i k \eta_{1}}{\pi b} \frac{V_{1}^{+}(\alpha) P_{1}(b, \alpha)}{\chi_{1}(\alpha)}+V_{1}^{+}(\alpha) P_{1}(a, \alpha) \\
\quad-\sum_{m=1}^{\infty} \frac{V_{1}^{+}\left(\beta_{m}^{(1)}\right) e^{i \beta_{m}^{(1)}} S_{1}^{+}\left(\beta_{m}^{(1)}\right)}{V_{1}^{\prime}\left(\beta_{m}^{(1)}\right)\left(\beta_{m}^{(1)}-\alpha\right)}-\frac{V_{1}^{+}(\alpha) e^{i \alpha l} S_{1}^{+}(\alpha)}{V_{1}(\alpha)} \\
=k \eta_{1} \frac{1}{(k+\alpha) V_{1}^{+}(k)}
\end{aligned}
$$

$$
\begin{aligned}
V_{2}^{+}(\alpha) & P_{1}(b, \alpha)+\frac{2 i k \eta_{2}}{\pi a} \sum_{m=1}^{\infty} \frac{V_{2}^{+}\left(\beta_{m}^{(2)}\right) P_{1}\left(a, \beta_{m}^{(2)}\right)}{X_{2}^{\prime}\left(\beta_{m}^{(2)}\right)\left(\beta_{m}^{(2)}-\alpha\right)}+\frac{2 i k \eta_{2}}{\pi a} \frac{V_{2}^{+}(\alpha) P_{1}(a, \alpha)}{X_{2}(\alpha)} \\
& -\sum_{m=1}^{\infty} \frac{V_{2}^{+}(\alpha)\left(\beta_{m}^{(2)}\right) e^{i \beta_{m}^{(2)}} S_{2}^{+}\left(\beta_{m}^{(2)}\right)}{V_{2}^{\prime}\left(\beta_{m}^{(2)}\right)\left(\beta_{m}^{(2)}-\alpha\right)}-\frac{V_{2}^{+}(\alpha) e^{i \alpha l} S_{2}^{+}(a, \alpha)}{V_{2}(\alpha)} \\
= & k \eta_{2} \frac{1}{(k+\alpha) V_{2}^{+}(k)},
\end{aligned}
$$




$$
\begin{aligned}
& e^{-i \alpha l} V_{1}^{-}(\alpha) P_{1}(a, \alpha)-\frac{2 i k \eta_{1}}{\pi b} \sum_{m=1}^{\infty} \frac{V_{1}^{+}\left(\beta_{m}^{(1)}\right) e^{i \beta_{m}^{(1)} l} P_{1}\left(b,-\beta_{m}^{(1)}\right)}{x_{1}^{\prime}\left(\beta_{m}^{(1)}\right)\left(\beta_{m}^{(1)}+\alpha\right)} \\
& -\frac{2 i k \eta_{1}}{\pi b} \frac{V_{1}^{-}(\alpha) e^{-i \alpha l} P_{1}(b, \alpha)}{\chi_{1}(\alpha)}-\sum_{m=1}^{\infty} \frac{V_{1}^{+}\left(\beta_{m}^{(1)}\right) e^{i \beta_{m}^{(1)} l} R_{1 *}^{-}\left(-\beta_{m}^{(1)}\right)}{V_{1}^{\prime}\left(\beta_{m}^{(1)}\right)\left(\beta_{m}^{(1)}+\alpha\right)} \\
& -\frac{V_{1}^{-}(\alpha) e^{-i \alpha l} R_{1 *}^{-}(\alpha)}{V_{1}(\alpha)}=0, \\
& -e^{-i \alpha l} V_{2}^{-}(\alpha) P_{1}(b, \alpha)+\frac{2 i k \eta_{2}}{\pi a} \sum_{m=1}^{\infty} \frac{V_{2}^{+}\left(\beta_{m}^{(2)}\right) e^{i \beta_{m}^{(2)} l} P_{1}\left(a,-\beta_{m}^{(2)}\right)}{x_{2}^{\prime}\left(\beta_{m}^{(2)}\right)\left(\beta_{m}^{(2)}+\alpha\right)} \\
& +\frac{2 i k \eta_{2}}{\pi a} \frac{V_{2}^{-}(\alpha) e^{-i \alpha l} P_{1}(a, \alpha)}{x_{2}(\alpha)}+\sum_{m=1}^{\infty} \frac{V_{2}^{+}\left(\beta_{m}^{(2)}\right) e^{i \beta_{m}^{(2)} l} R_{2 *}^{-}\left(-\beta_{m}^{(2)}\right)}{V_{2}^{\prime}\left(\beta_{m}^{(2)}\right)\left(\beta_{m}^{(2)}+\alpha\right)} \\
& -\frac{V_{2}^{-}(\alpha) e^{-i \alpha l} R_{2 *}^{-}(\alpha)}{V_{2}(\alpha)}=0 .
\end{aligned}
$$

Here the dash (I) denotes the derivative with respect to $\alpha: V_{1,2}^{\prime}\left(\beta_{m}^{(1,2)}\right)=\left.(\partial / \partial \alpha) V_{1,2}(\alpha)\right|_{\alpha=\beta_{m}^{(1,2)}}$.

The formal solution of the coupled system of Wiener-Hopf equations given by (2.18a)-(2.18h) involves 16 unknown functions, namely, $P_{1}\left(a, \beta_{m}^{(1)}\right), P_{1}\left(a, \beta_{m}^{(2)}\right), P_{1}\left(a,-\beta_{m}^{(1)}\right), P_{1}\left(a,-\beta_{m}^{(2)}\right), P_{1}\left(b, \beta_{m}^{(1)}\right), P_{1}\left(b, \beta_{m}^{(2)}\right), P_{1}\left(b,-\beta_{m}^{(1)}\right), P_{1}\left(b,-\beta_{m}^{(2)}\right)$, $S_{1}^{+}\left(\beta_{m}^{(1)}\right), S_{1}^{+}\left(\beta_{m}^{(2)}\right), S_{2}^{+}\left(\beta_{m}^{(1)}\right), S_{2}^{+}\left(\beta_{m}^{(2)}\right), R_{1 *}^{-}\left(-\beta_{m}^{(1)}\right), R_{1 *}^{-}\left(-\beta_{m}^{(2)}\right), R_{2 *}^{-}\left(-\beta_{m}^{(1)}\right)$, and $R_{2 *}^{-}\left(-\beta_{m}^{(2)}\right)$. In order to determine them we substitute $\alpha=-\beta_{n}^{(1)},-\beta_{n}^{(2)}, n=1,2, \ldots$ in (2.18a), (2.18b), (2.18g), (2.18h) and $\alpha=\beta_{n}^{(1)}, \beta_{n}^{(2)}, n=1,2, \ldots$ in (2.18c), (2.18d), (2.18e), (2.18f), respectively. So, at a first sight, we get 16 infinite systems of algebraic equations. After some straightforward but tedious algebraic manipulations, one can reduce the number of the unkown sets to 8, namely, $P_{1}\left(a, \pm \beta_{m}^{(2)}\right), P_{1}\left(b, \pm \beta_{m}^{(1)}\right), R_{1 *}^{-}\left(-\beta_{m}^{(1)}\right), R_{1 *}^{-}\left(-\beta_{m}^{(2)}\right), R_{2 *}^{-}\left(-\beta_{m}^{(1)}\right)$ and $R_{2 *}^{-}\left(-\beta_{m}^{(2)}\right)$. These coupled systems of algebraic equations will be solved numerically. The approach used in solving the infinite system of algebraic equations is similar to that employed by Rawlins [20]. By using the edge conditions and the asymptotic behavior of $\beta_{n}^{(1)}$ and $\beta_{n}^{(2)}$, one can show that the convergence of the infinite series appearing in these equations is rapid enough to allow truncation at, say $N$. Consequently the infinite systems are replaced by the corresponding finite systems of $8 N \times 8 N$ algebraic equations and then solved by standard numerical algorithms. The value of $N$ was increased until the reflected field amplitude being calculated did not change in a given number of decimal places. A typical result is provided by Figure 3. It can be seen that the reflected field amplitude becomes insensitive to the increase of the truncation number for $N>6$. 


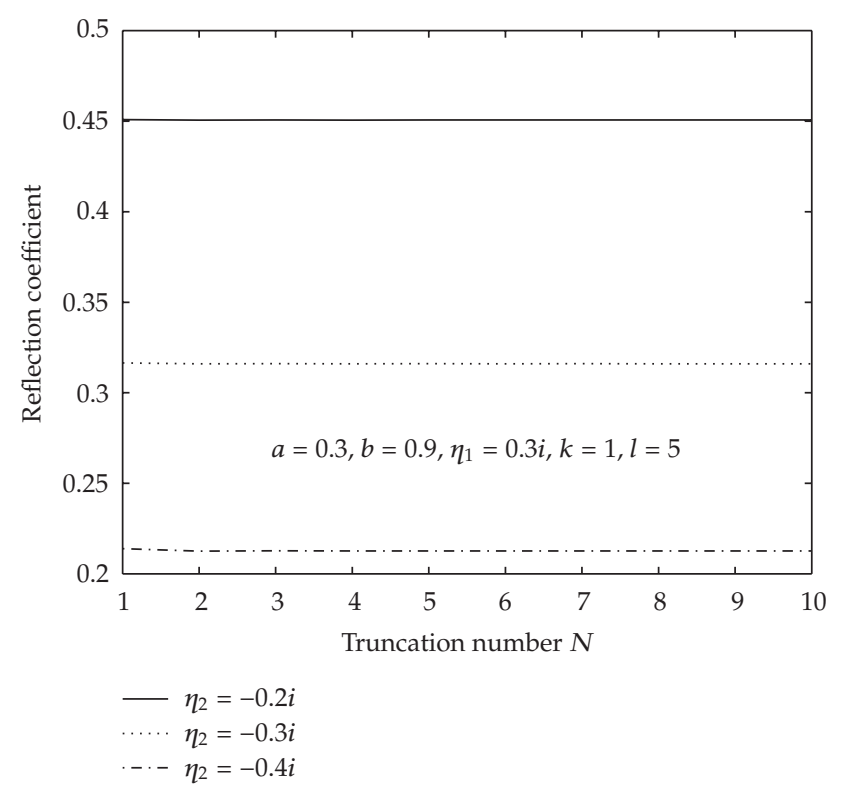

Figure 3: Reflected field amplitude versus the truncation number.

\section{Scattered Field}

For $\rho \in(a, b)$ and $z<0$, the reflected wave propagating backward is obtained by taking the inverse Fourier transform of $F_{-}(\rho, \alpha)$, namely,

$$
\begin{gathered}
u_{1}(\rho, \alpha)=\frac{1}{2 \pi} \int_{-\infty}^{\infty}\left(\frac{b T_{2}(\rho, b, \alpha) P_{1}(a, \alpha)-a T_{2}(\rho, a, \alpha) P_{1}(b, \alpha)}{a b T_{1}(a, b, \alpha)}\right. \\
\left.-F_{1}(\rho, \alpha)-e^{i \alpha l} F_{+}(\rho, \alpha)\right) e^{-i \alpha z} d \alpha .
\end{gathered}
$$

The above integral is calculated by closing the contour in the upper half plane and evaluating the residue contributions from the simple poles occurring at the zeros of $T_{1}(a, b, \alpha)$ lying in the upper $\alpha$-half-plane. The reflection coefficient $R$ of the fundamental mode is defined as the ratio of reflected and incident waves amplitudes. It is computed from the contribution of the first pole at $\alpha=k$. The result is

$$
\mathcal{R}=-\frac{i}{2 a b k \log (b / a)}\left[b P_{1}(a, k)-a P_{1}(b, k)\right]
$$

Similarly, the transmitted field in the region $\rho \in(a, b)$ and $z>l$ is obtained by inverting $F_{+}(\rho, \alpha)$ :

$u_{1}(\rho, \alpha)=\frac{1}{2 \pi} \int_{-\infty}^{\infty}\left(\frac{b T_{2}(\rho, b, \alpha) P_{1}(a, \alpha)-a T_{2}(\rho, a, \alpha) P_{1}(b, \alpha)}{a b T_{1}(a, b, \alpha)}-F_{-}(\rho, \alpha)-F_{1}(\rho, \alpha)\right) e^{-i \alpha z} d \alpha$ 
The transmission coefficient $\tau$ of the fundamental mode, which is defined as the ratio of the amplitudes of the transmitted and incident waves, is obtained by evaluating the integral in (3.3) for $z>l$. This integral is now computed by closing the contour in the lower half of the complex $\alpha$-plane. The pole of interest is at $\alpha=-k$ whose contribution gives

$$
-\frac{i}{2 a b k \log (b / a)}\left[b P_{1}(a,-k)-a P_{1}(b,-k)\right] \frac{e^{i k z}}{\rho}
$$

with

$$
\begin{aligned}
{\left[b P_{1}(a,-k)-a P_{1}(b,-k)\right]=} & \frac{2 a b k \log (b / a)}{i} \\
& -\frac{e^{-i k l}}{V_{1}^{+}(k)}\left\{\frac{2 i k \eta_{1}}{\pi} \sum_{m=1}^{\infty}\left(\frac{V_{1}^{+}\left(\beta_{m}^{(1)}\right) e^{i \beta_{m}^{(1)} l} P_{1}\left(b,-\beta_{m}^{(1)}\right)}{x_{1}^{\prime}\left(\beta_{m}^{(1)}\right)\left(\beta_{m}^{(1)}-k\right)}\right)\right. \\
& \left.+b \sum_{m=1}^{\infty}\left(\frac{V_{1}^{+}\left(\beta_{m}^{(1)}\right) e^{i \beta_{m}^{(1)} l} R_{1 *}^{-}\left(-\beta_{m}^{(1)}\right)}{V_{1}^{\prime}\left(\beta_{m}^{(1)}\right)\left(\beta_{m}^{(1)}-k\right)}\right)\right\},
\end{aligned}
$$

or equivalently

$$
\begin{aligned}
{\left[b P_{1}(a,-k)-a P_{1}(b,-k)\right]=} & \frac{2 a b k \log (b / a)}{i} \\
& -\frac{e^{-i k l}}{V_{2}^{+}(k)}\left\{-\frac{2 i k \eta_{2}}{\pi} \sum_{m=1}^{\infty}\left(\frac{V_{2}^{+}\left(\beta_{m}^{(2)}\right) e^{i \beta_{m}^{(2)} l} P_{1}\left(a,-\beta_{m}^{(2)}\right)}{x_{2}^{\prime}\left(\beta_{m}^{(2)}\right)\left(\beta_{m}^{(2)}-k\right)}\right)\right. \\
& \left.+a \sum_{m=1}^{\infty}\left(\frac{V_{2}^{+}\left(\beta_{m}^{(2)}\right) e^{i \beta_{m}^{(2)} l} R_{2 *}^{-}\left(-\beta_{m}^{(2)}\right)}{V_{2}^{\prime}\left(\beta_{m}^{(2)}\right)\left(\beta_{m}^{(2)}-k\right)}\right)\right\}
\end{aligned}
$$

The first term in (3.4a) or in (3.4b) cancels out the incident fundamental mode as expected. The transmission coefficient is then given by

$$
\tau=1-\frac{i}{2 a b k \log (b / a)}\left[b P_{1}(a,-k)-a P_{1}(b,-k)\right]
$$

\section{Computational Results}

In order to observe the influence of the different parameters such as the surface impedances $\left(\eta_{1}\right.$ and $\left.\eta_{2}\right)$, the width $(l)$ of the impedance loadings, and the distance $b-a$ between the two coaxial cylinders on the reflection coefficient, some numerical results are presented in this section. In what follows the impedances are assumed to be purely reactive. 


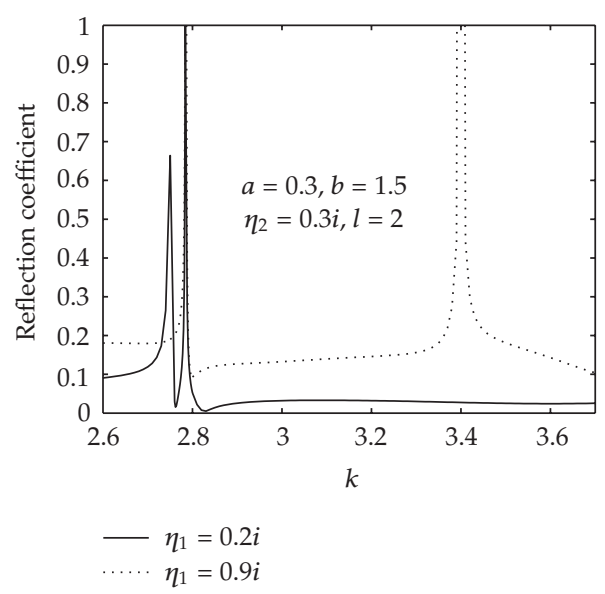

(a)

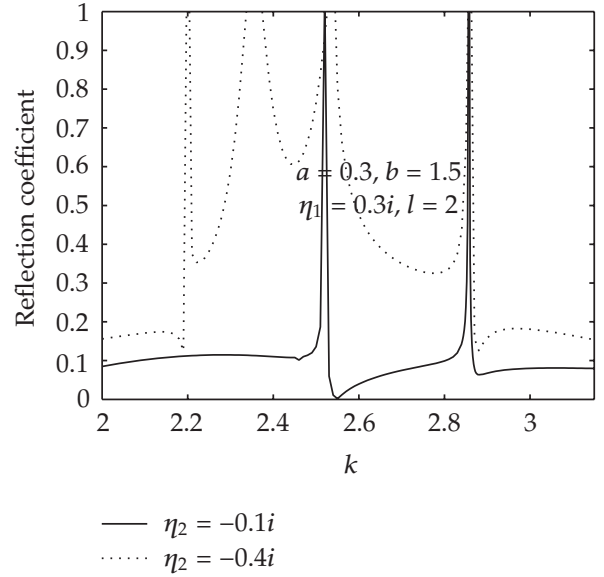

(b)

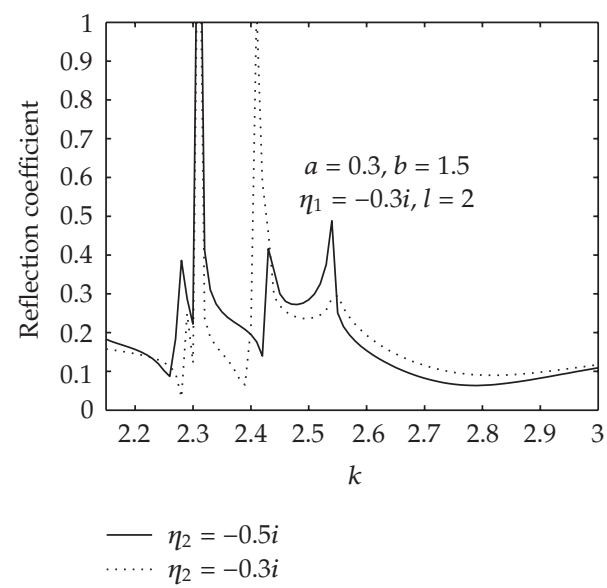

(c)

Figure 4: (a) Amplitude of the reflection coefficient versus the frequency, for different values of the impedance loading on the inner conductor (the case where $\eta_{1}$ and $\eta_{2}$ are capacitive). (b) Amplitude of the reflection coefficient versus the frequency, for different values of the impedance loading on the inner conductor (the case where $\eta_{1}$ is capacitive and $\eta_{2}$ is inductive ). (c) Amplitude of the reflection coefficient versus the frequency, for different values of the impedance loading on the inner conductor (the case where $\eta_{1}$ and $\eta_{2}$ are inductive).

Figures $4(a)-4(c)$ show the amplitude of the reflection coefficient versus the frequency for different values of $\eta_{2}$ while $\eta_{1}, a, b$, and $l$ are held fixed. If $\eta_{1}$ and $\eta_{2}$ are both capacitive, the band-stop frequencies of the configuration are shifted to the right for increasing values of $\left|\eta_{2}\right|$ (Figure 4(a)). In the case where $\eta_{1}$ is capacitive while $\eta_{2}$ is inductive, the band stop characteristic of the configuration is observed at lower frequencies for increasing the values of $\left|\eta_{2}\right|$ (Figure 4(b)). Finally, Figure 4(c) displays the case where the both surface impedances are inductive.

Figure 5 depicts the variation of the reflected field amplitude for different values of distance between the coaxial cylinders. It is observed that the amplitude of the reflected field decreases when the separation distance $b-a$ increases, as expected. Furthermore, for 


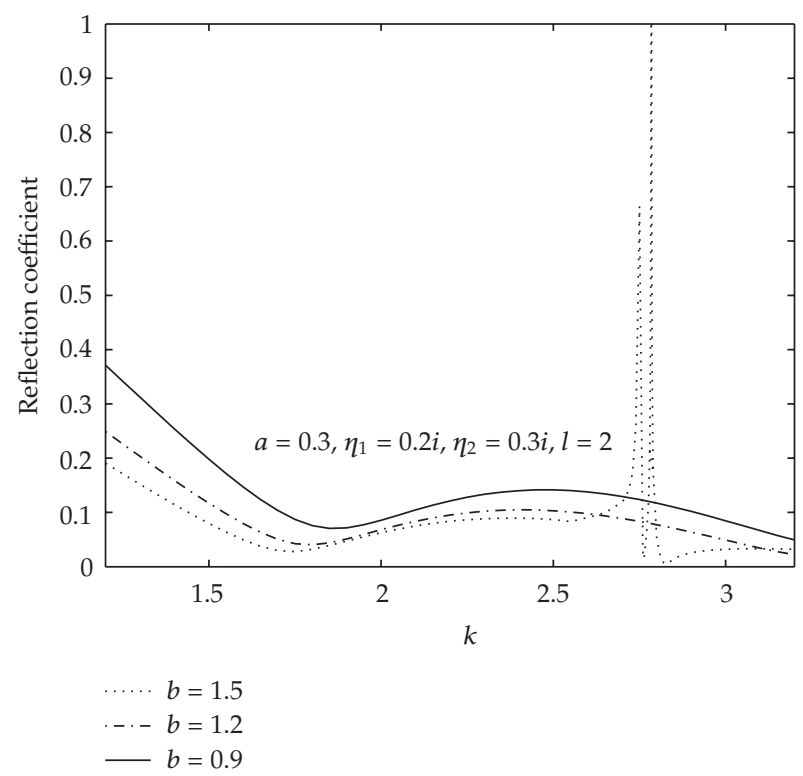

Figure 5: Amplitude of the reflection coefficient versus the frequency for different values of the outer cylinder radius $b$.

decreasing values of the outer cylinder radius, the band-stop frequencies of the configuration are shifted to the right. This means that total reflections occur at higher frequencies if the radius of the outer cylinder decreases.

The effect of the width for the impedance loadings on the reflection coefficient is shown in Figure 6. The number of resonances corresponding to the variation of the reflected field increases when the value of $l$ increases. But the amplitude related to the reflected field is not affected too much by the width of the impedance surfaces. It is also seen that the curves related to reflected field amplitude approaches to the one calculated from (5.2a), when $k l \gg 1$.

\section{Discussion}

When we let $l \rightarrow \infty$, the Wiener- Hopf equations in (2.10) reduce to

$$
\begin{aligned}
i k a \Psi_{1}^{-}(a, \alpha)+V_{1}(\alpha) P_{1}(a, \alpha) & =\frac{2 i k \eta_{1}}{\pi b T_{1}(a, b, \alpha)} P_{1}(b, \alpha)+\frac{k \eta_{1}}{k+\alpha} \\
i k b \Psi_{2}^{-}(b, \alpha)-V_{2}(\alpha) P_{1}(b, \alpha) & =\frac{-2 i k \eta_{2}}{\pi a T_{1}(a, b, \alpha)} P_{1}(a, \alpha)+\frac{k \eta_{2}}{k+\alpha}
\end{aligned}
$$

Then, the reflection coefficient in (3.2) takes the following form:

$$
\mathcal{R}=\frac{-i}{2 a b k \log (b / a) V_{1}^{+}(k)}\left[\frac{2 i k \eta_{1}}{\pi} \sum_{m=1}^{\infty} \frac{V_{1}^{+}\left(\beta_{m}^{(1)}\right) P_{1}\left(b, \beta_{m}^{(1)}\right)}{x_{1}^{\prime}\left(\beta_{m}^{(1)}\right)\left(\beta_{m}^{(1)}-k\right)}+\frac{b \eta_{1}}{2 V_{1}^{+}(k)}\right]
$$




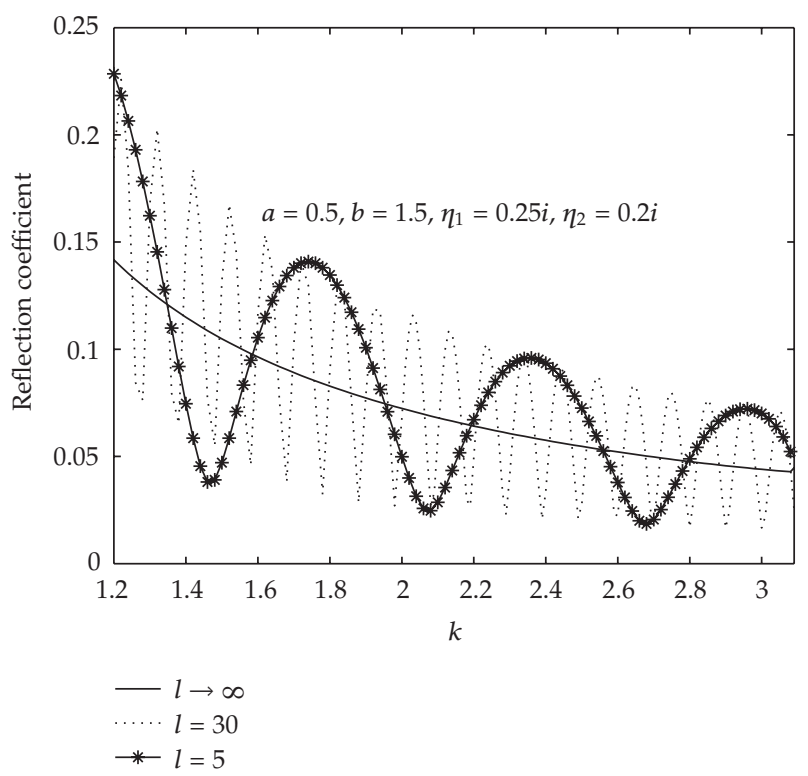

Figure 6: Amplitude of the reflection coefficient versus the frequency for different impedance loadings length $l$.

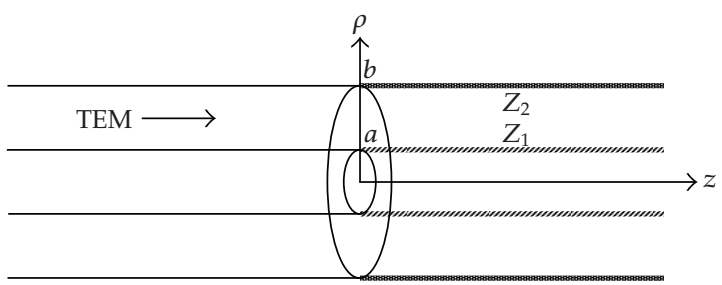

Figure 7: Junction of perfectly conducting and impedance loaded coaxial waveguides $(l \rightarrow \infty)$.

or

$$
\mathcal{R}=\frac{-i}{2 a b k \log (b / a) V_{2}^{+}(k)}\left[-\frac{2 i k \eta_{2}}{\pi} \sum_{m=1}^{\infty} \frac{V_{2}^{+}\left(\beta_{m}^{(2)}\right) P_{1}\left(a, \beta_{m}^{(2)}\right)}{\chi_{2}^{\prime}\left(\beta_{m}^{(2)}\right)\left(\beta_{m}^{(2)}-k\right)}+\frac{a \eta_{2}}{2 V_{2}^{+}(k)}\right]
$$

which is nothing but the results related to the junction of perfectly conducting and impedance coaxial waveguides shown in Figure 7.

Equations (5.2a) and (5.2b) are obtained by using the Wiener-Hopf equations in (5.1a) and $(5.1 b)$, respectively. It can be checked easily that the two expressions are equivalent. 


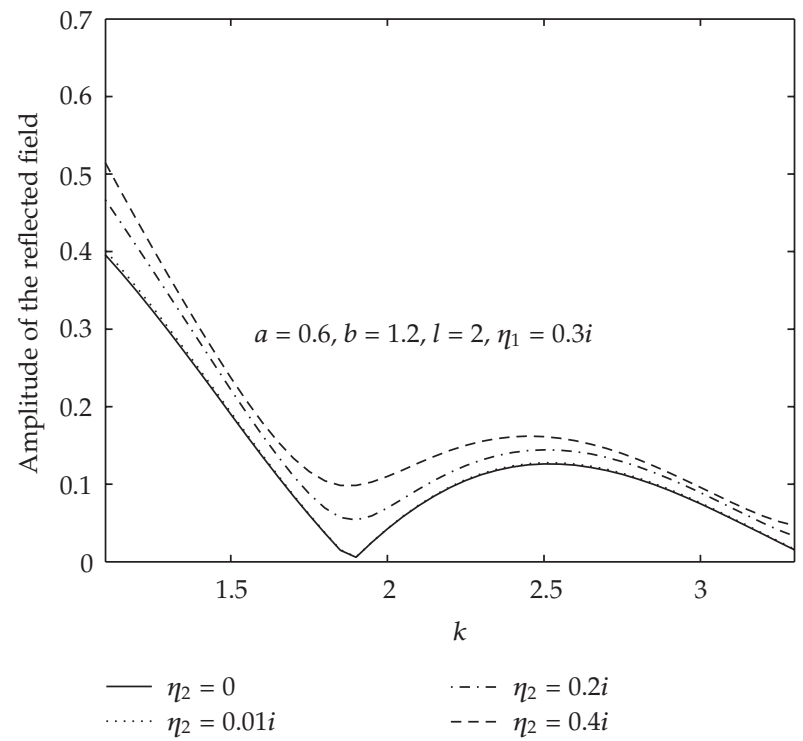

Figure 8: Amplitude of the reflection coefficient versus the frequency for different values of $\eta_{2}$.

$P_{1}\left(b, \beta_{m}^{(1)}\right)$ and $P_{1}\left(a, \beta_{m}^{(2)}\right)$ appearing in the above expressions are to be determined through the solution of the following 2 sets of infinite systems of algebraic equations:

$$
\begin{aligned}
V_{1}^{+}\left(\beta_{n}^{(2)}\right) P_{1}\left(a, \beta_{n}^{(2)}\right)+\frac{4 k^{2} \eta_{1} \eta_{2}}{\pi^{2} a b} \frac{V_{1}^{+}\left(\beta_{n}^{(2)}\right)}{x_{1}\left(\beta_{n}^{(2)}\right) V_{2}^{+}\left(\beta_{n}^{(2)}\right)} \sum_{m=1}^{\infty}\left(\frac{V_{2}^{+}\left(\beta_{m}^{(2)}\right) P_{1}\left(a, \beta_{m}^{(2)}\right)}{x_{2}^{\prime}\left(\beta_{m}^{(2)}\right)\left(\beta_{m}^{(2)}-\beta_{n}^{(2)}\right)}\right) \\
\quad-\frac{2 i k \eta_{1}}{\pi b} \sum_{m=1}^{\infty}\left(\frac{V_{1}^{+}\left(\beta_{m}^{(1)}\right) P_{1}\left(b, \beta_{m}^{(1)}\right)}{x_{1}^{\prime}\left(\beta_{m}^{(1)}\right)\left(\beta_{m}^{(1)}-\beta_{n}^{(2)}\right)}\right) \\
=\frac{k \eta_{1}}{\left(k+\beta_{n}^{(2)}\right) V_{1}^{+}(k)}-\frac{2 i k^{2} \eta_{1} \eta_{2} V_{1}^{+}\left(\beta_{n}^{(2)}\right)}{\left(k+\beta_{n}^{(2)}\right) x_{1}\left(\beta_{n}^{(2)}\right) V_{2}^{+}\left(\beta_{n}^{(2)}\right) V_{2}^{+}(k)}, \\
-V_{2}^{+}\left(\beta_{n}^{(1)}\right) P_{1}\left(b, \beta_{n}^{(1)}\right)-\frac{4 k^{2} \eta_{1} \eta_{2}}{\pi^{2} a b} \frac{V_{2}^{+}\left(\beta_{n}^{(1)}\right)}{x_{2}\left(\beta_{n}^{(1)}\right) V_{1}^{+}\left(\beta_{n}^{(1)}\right)} \sum_{m=1}^{\infty} \frac{V_{1}^{+}\left(\beta_{m}^{(1)}\right) P_{1}\left(b, \beta_{m}^{(1)}\right)}{x_{1}^{\prime}\left(\beta_{m}^{(1)}\right)\left(\beta_{m}^{(1)}-\beta_{n}^{(1)}\right)} \\
\quad+\frac{2 i k \eta_{2}}{\pi a} \sum_{m=1}^{\infty}\left(\frac{V_{2}^{+}\left(\beta_{m}^{(2)}\right) P_{1}\left(a, \beta_{m}^{(2)}\right)}{x_{2}^{\prime}\left(\beta_{m}^{(2)}\right)\left(\beta_{m}^{(2)}-\beta_{n}^{(1)}\right)}\right) \\
=\frac{2 i k^{2} \eta_{1} \eta_{2} V_{2}^{+}\left(\beta_{n}^{(1)}\right)}{\left(k+\beta_{n}^{(1)}\right) V_{2}^{+}(k)}-\frac{\left.\beta_{n}^{(1)}\right) x_{2}\left(\beta_{n}^{(1)}\right) V_{1}^{+}\left(\beta_{n}^{(1)}\right) V_{1}^{+}(k)}{\left(k+\beta_{n}^{(2)}\right.} .
\end{aligned}
$$


Finally, for $\left|\eta_{2}\right| \rightarrow 0$, the results obtained in the present work to that previously obtained in [3]. Indeed, from Figure 8 we can see that for decreasing values of $\left|\eta_{2}\right|$, the curves approach the result related to the finite-length impedance loading in the outer conductor of a coaxial waveguide obtained in [3] (solid line in Figure 8). This can be considered as a check of the analysis made in this paper.

\section{Conclusion}

In the present work the propagation of TEM wave in a coaxial waveguide with finite-length impedance loading is investigated rigorously through the Wiener-Hopf technique. In order to obtain the explicit expressions of the reflection coefficient, the problem is first reduced into two coupled modified Wiener-Hopf equations and then solved exactly in a formal sense by using the factorization and decomposition procedures. The formal solution involves infinite series with 8 sets of unknown coefficients satisfying 8 infinite sets of algebraic equations which are solved numerically. The advantage of the present method is that the solution obtained here is valid for all frequencies and impedance lengths. Furthermore, it is observed that for certain values of the surface impedances full reflection occurs, showing that this configuration may be used as a band-stop filter.

Finally, it is noteworthy that the Weiner-Hopf solution provided here could be extended to treat the case where the lengths of the impedances on the inner and outer conductors are different. Other future work could lie in the investigation of wave propagation in coaxial waveguides with successive finite-length impedance loadings.

\section{References}

[1] R. F. Huang and D. M. Zhang, "Application of mode matching method to analysis of axisymmetrical coaxial discontinuity structures used in permeability and/or permittivity measurement," Progress in Electromagnetics Research, vol. 67, pp. 205-230, 2007.

[2] N.-E. Belhadj-Tahar and A. Fourrier-Lamer, "Broad-band analysis of a coaxial discontinuity used for dielectric measurements," IEEE Transactions on Microwave Theory and Techniques, vol. 34, no. 3, pp. 346-350, 1986.

[3] N.-E. Belhadj-Tahar, A. Fourrier-Lamer, and H. de Chanterac, "Broad-band simultaneous measurement of complex permittivity and permeability using a coaxial discontinuity," IEEE Transactions on Microwave Theory and Techniques, vol. 38, no. 1, pp. 1-7, 1990.

[4] P. I. Somlo, "The computation of coaxial line step capacitances," IEEE Transactions on Microwave Theory and Techniques, vol. 15, pp. 48-53, 1967.

[5] W. K. Gwarek, "Computer-aided analysis of arbitrarily shaped coaxial discontinuities," IEEE Transactions on Microwave Theory and Techniques, vol. 36, no. 2, pp. 337-342, 1988.

[6] A. P. Orfanidis, G. A. Kyriacou, and J. N. Sahalos, "A mode-matching technique for the study of circular and coaxial waveguide discontinu- ities based on closed-form coupling integrals," IEEE Transactions on Microwave Theory and Techniques, vol. 48, pp. 880-883, 2000.

[7] W. Yu, R. Mittra, and S. Dey, "Application of the nonuniform FDTD technique to analysis of coaxial discontinuity structures," IEEE Transactions on Microwave Theory and Techniques, vol. 49, no. 1, pp. 207209, 2001.

[8] S. Vijayaraghayan and R. K. Arora, "Scattering of a shielded surface wave in a coaxial waveguide by a wall impedance discontinuity," IEEE Transactions on Microwave Theory and Techniques, vol. 18, pp. 736-739, 1971.

[9] M. S. Bobrovnikov, "Diffraction of electromagnetic waves at a surface impedance discontinuity in a coaxial waveguide," Soviet Physics Journal, vol. 9, pp. 4-6, 1966.

[10] D. Varon, "Radial line band rejection filters in coaxial waveguides," IEEE Transactions on Microwave Theory and Techniques, vol. 15, no. 12, pp. 680-687, 1967. 
[11] L. A. Weinstein, The Theory of Diffraction and the Factorization Method, The Golem Press, Boulder, Colo, USA, 1969.

[12] T. B. A. Senior and J. L. Volakis, Approximate Boundary Conditions in Electromagnetics, IEE Press, London, UK, 1995.

[13] S. F. Mahmoud, Electromagnetic Waveguides: Theory and Applications, IEE Press, London, UK, 1991.

[14] F. Hacivelioğlu and A. Büyükaksoy, "Wiener-Hopf analysis of finite-length impedance loading in the outer conductor of a coaxial waveguide," Progress in Electromagnetics Research B, vol. 5, pp. 241-251, 2008.

[15] K. Kobayashi, "Modified Wiener-Hopf geometries," in Analytical and Numerical Methods in Electromagnetic Wave Theory, M. Hashimoto, M. Idemen, and O. A. Tretyakov, Eds., Science House, Tokyo, Japan, 1993.

[16] I. H. Tayyar, A. Büyükaksoy, and A. Işıkyer, "A Wiener-Hopf analysis of the parallel plate waveguide with finite length impedance loading," Radio Science, vol. 43, pp. 1-12, 2008.

[17] M. Idemen, "Confluent edge conditions for the electromagnetic wave at the edge of a wedge bounded by material sheets," Wave Motion, vol. 32, pp. 37-55, 2000.

[18] R. Mittra and S. W. Lee, Analytical Techniques in the Theory of Guided Waves, McMillan, New York, NY, USA, 1971.

[19] B. Noble, Methods Based on the Wiener-Hopf Technique for the Solution of Partial Differential Equations, vol. 7 of International Series of Monographs on Pure and Applied Mathematics, Pergamon Press, New York, NY, USA, 1958.

[20] A. D. Rawlins, "Radiation of sound from an unflanged rigid cylindrical duct with an acoustically absorbing internal surface," Proceedings of the Royal Society A, vol. 361, no. 1704, pp. 65-91, 1978. 


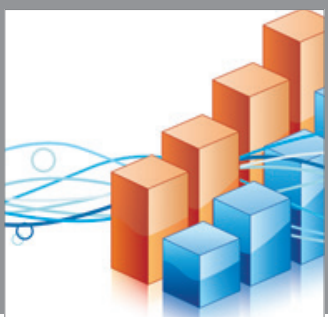

Advances in

Operations Research

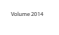

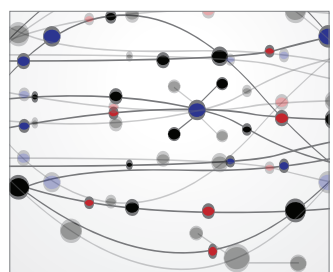

\section{The Scientific} World Journal
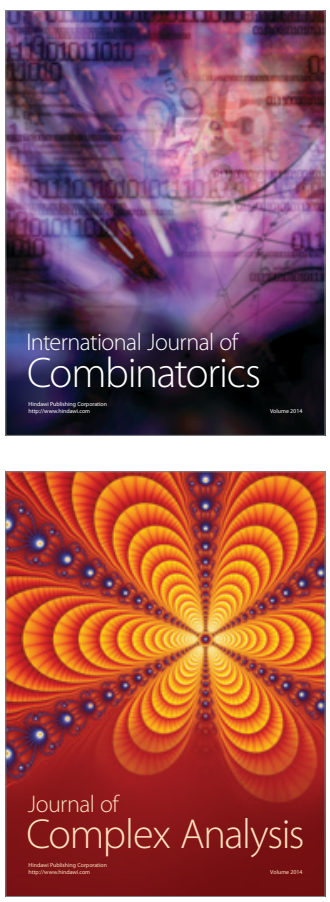

International Journal of

Mathematics and

Mathematical

Sciences
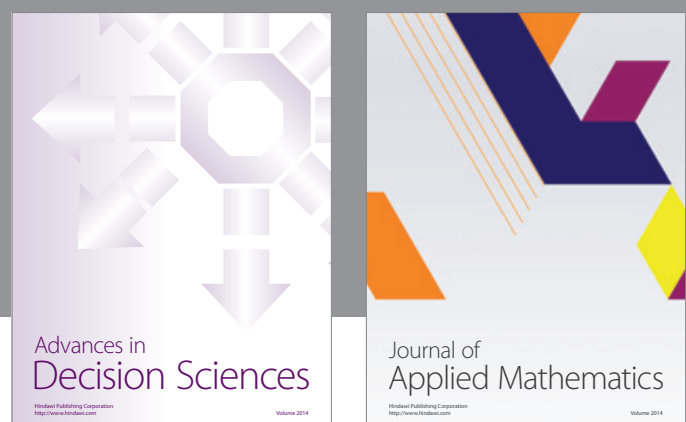

Journal of

Applied Mathematics
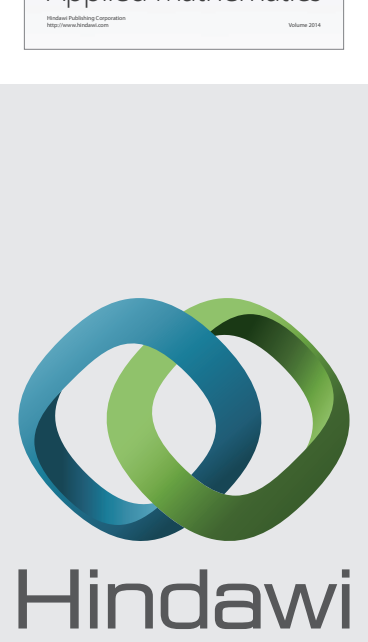

Submit your manuscripts at http://www.hindawi.com
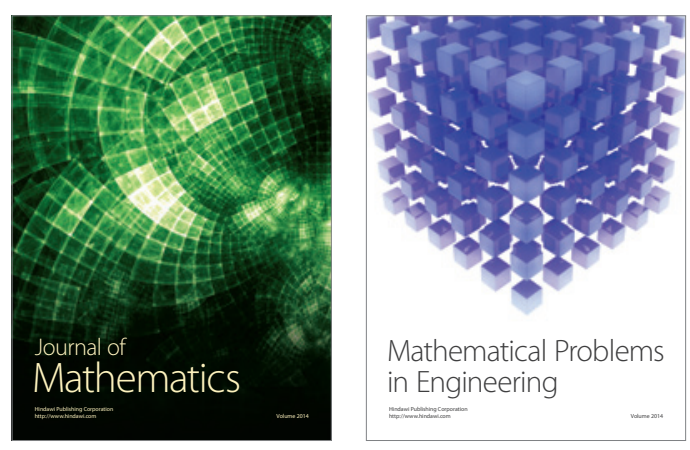

Mathematical Problems in Engineering
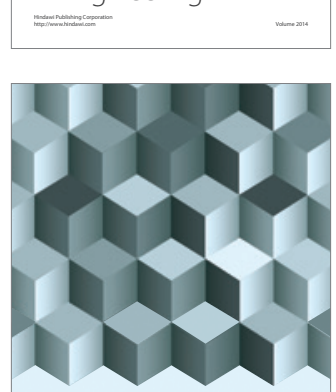

Journal of

Function Spaces
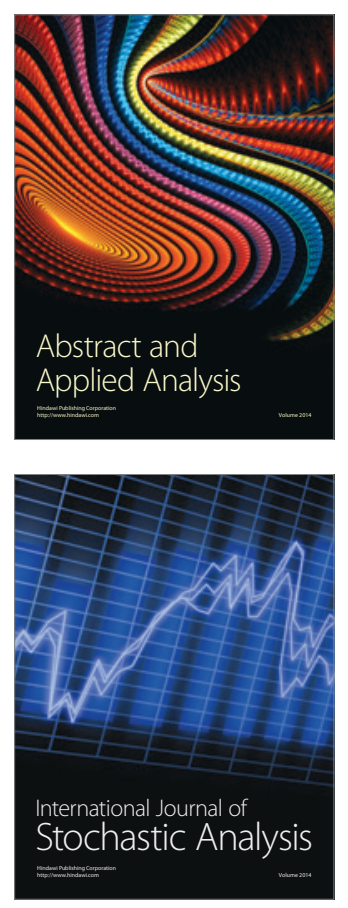

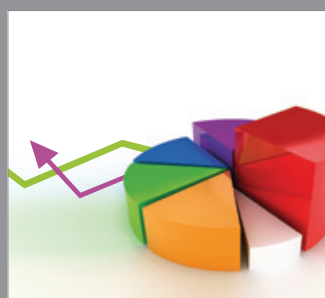

ournal of

Probability and Statistics

Promensencen
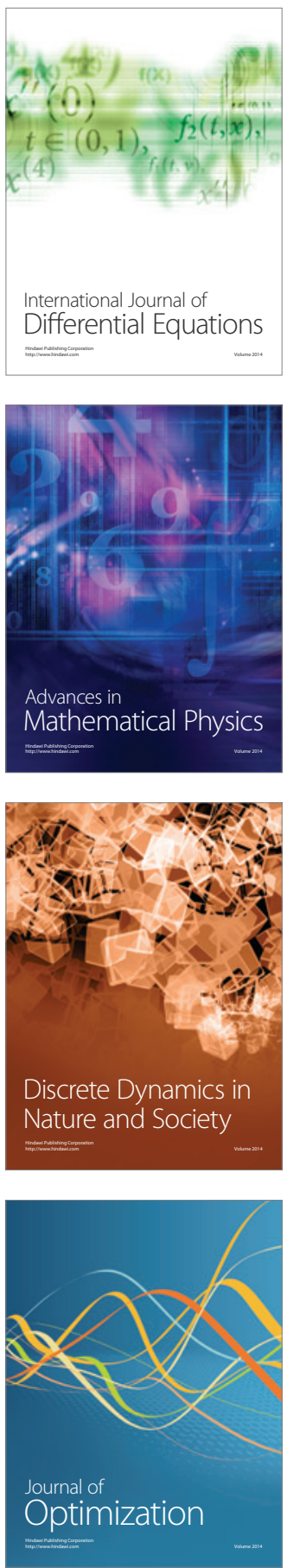